\title{
Biomechanical Evaluation of a New Anatomic Locking Buttress Plate for Posteromedial Tibial Plateau Fixation.
}

Kai Cheng Lin ( $\sim$ orthokcl@gmail.com)

Kaohsiung Veterans General Hospital

Yih Wen Tarng

Kaohsiung Veterans General Hospital

Hung-Wen Wei

National Yang-Ming Medical College: National Yang Ming Chiao Tung University - Yangming Campus

Kun-Jhih Lin

Chung Yuan Christian University

\section{Research Article}

Keywords: Finite element methods, Locking plate, Tibial plateau, Posteromedial fragment

Posted Date: July 6th, 2021

DOI: https://doi.org/10.21203/rs.3.rs-631817/v1

License: (c) (i) This work is licensed under a Creative Commons Attribution 4.0 International License. Read Full License 


\section{Abstract}

Background: Dealing with high-energy fractures of the tibial plateau remains a challenge despite advances in implants, surgical approaches, and imaging methods. Posterior buttress plate is most commonly used implant but the fixation stability is still a challenge. Recently, a newly designed tibial locking plate was introduced that aims to provide better fixation strength for tibial plateau split fracture. This study compared the biomechanical strength of three different posteromedial tibial plateau split fracture fixation methods.

Methods: The tibial plateau fractures were simulated using a human tibiae model. Each fracture model was virtually implanted with one of the three following constructs, proximal medial tibial plate (PMT), proximal posterior medial tibial plate (PPMT), and posterior T-shaped buttress plate (TBP). Posteromedial fragment vertical subsidence was measured under $2000 \mathrm{~N}$ joint contact force. The maximum Equivalent stress on the bone plate and bone screw and the construct stiffness were determined.

Results: The proximal medial tibial plate (PMT) allowed the least posteromedial fragment subsidence and produced higher construct stiffness than each of the other two constructs. However, the proximal posterior medial tibial plate (PPMT) showed higher stiffness than the T-shaped buttress plate (TBP). The maximum Equivalent stress was the smallest for the proximal medial tibial plate (PMT).

Conclusion: This study showed that the proximal medial tibial locking plate or proximal posterior medial tibial locking plate were biomechanically more stable fixation methods for posteromedial split tibial plateau fractures.

\section{Introduction}

A posteromedial tibial split fracture is a notable medial plateau injury pattern. The fracture line appears in the coronal plane with a separate posteromedial osteoarticular fragment of variable size. Clinically, ignoring this fragment can lead to distal displacement, with posterior medial femoral condyle subluxation ${ }^{1}$. Bone plate fixation is commonly employed to fix the fragment bone.

For several years, posteromedial implant popularity has gained some attraction but most surgeons will simply apply the conventional $3.5 \mathrm{~mm}$ T-plate. Due to insufficient implant stability, bone nonunion sometimes occurs even from direct fixation. Moreover, reduction loss and fixation loss from indirect fixation and insufficient fixation have also occurred. Therefore, a more rigid implant that provides enough stability to initiate bone union should not be overlooked.

A specialized anatomical locking plate (Proximal Posterior Medial Tibia Locking Plate) that provides sufficient stability for posteromedial tibial plateau fracture fixation was designed. This innovative implant is specific to posteromedial coronal fragment osteosynthesis with a locking mechanism. The features of this implant include: 1 . Anatomical design that does not require plate bending for fitting. 2. A 
conventional hole is located on the posteromedial fracture spike to allow buttressing. 3. Locking designs for angular stability. 4. The plate can be placed juxta-articular for subchondral fixation.

The purpose of the study is: (i) to describe the details of an innovative anatomical locking plate for the posteromedial tibial plateau; and (ii) to evaluate the safety and fixation efficacy of this innovative plate. We used finite element analysis to provide adequate information on this implant stability. By comparing it to other fixation methods, we propose this implant as a possible option for this surgery.

\section{Methods}

\section{Model preparation}

The three-dimensional lower leg finite element model included the tibia. The bony structures were generated using a computed topography data set segmentation from the Visible Human Project ${ }^{2}$. The 3D tibia model was reconstructed via the cortical shell and cancellous core. The fracture model was made based on the fracture morphology described by Higgins ${ }^{1}$. Three different proximal tibial plate designs were employed in this simulation; the Proximal Posterior Medial Tibia Locking Plate (PPMT), Proximal Medial Tibia Locking Plate (PMT) (A Plus Biotechnology Co. Ltd., New Taipei City, Taiwan) and the conventional T-shaped Buttress Plate (TBP) (Depuy Synthes, Paoli, PA). Three .5-mm locking screws were used to affix the proximal tibia plate. The screw placements for each plate fixation are shown in Fig. 1.

\section{Implant}

All three models were meshed using ANSYS Workbench (ANSYS Inc., Canonsburg, PA, United States). The cortical bone and cancellous bone interface was bonded. The screws were bonded to the corresponding bony structure. The screw heads were bonded to the corresponding screw hole on the bone plate. The bone-plate interface was friction. The friction coefficient between the bone-plate interactions was $0.42^{3}$. The material properties for the bony structure (cortical bone, Young's modulus: $15.1 \mathrm{GPa}$, Poisson's ratio: 0.3; cancellous bone, Young's modulus: $100 \mathrm{MPa}$, Poisson's ratio: 0.3 ) and metallic implants (titanium, Young's modulus: $110 \mathrm{GPa}$, Poisson's ratio: 0.3$)^{3}$ were assigned accordingly.

The distal end of the tibia was fully fixed in all degrees of freedom for the load and boundary conditions in each group. Two thousand newton forces were applied to the proximal tibial plateau to simulate the single leg stand. The knee contact force is not uniformly shared between the tibia condyles. The compression load was divided into $60 \%$ applied to the medial side and $40 \%$ to the lateral side, as in previous studies (Fig. 2).

\section{Evaluating parameters}

Each group was compared in terms of the maximum von Mises stress in each plate, screw and tibia bone. The plate-bone construct stiffness was calculated. The construct stiffness was derived from the load and vertical displacement data. 


\section{Results}

For the bone plate equivalent stress, the stress patterns and values on the bone plates in all models are shown in Fig. 3. The maximum PPMT plate stress (106.13 MPa) is slightly higher than PMT (87.10 MPa) and TBP plates (93.68 MPa). The PPMT plate stress distribution is similar to that of the TBP plate. The stress concentration was found around the distal end of the thread hole. The PMT stress is distributed around the distal end of the thread hole. The PPMT stress is distributed on the distal end of the plate around the sliding hole. The TBP stress is distributed around the screw hole. The bone screw stress patterns and values in all models are shown in Fig. 4. The maximum stress was 297.22 MPa in PPMT plate, 205.53 MPa in PMT plate, and 193.08 MPa in TBP plate, respectively. The maximum bone screw stress in each group occurred on the distal end of the screw (Fig. 5). The opposite result was observed on the bone fragment. The maximum PPMT plate stress $(27.04 \mathrm{MPa})$ is slightly inferior with PMT (31.40 $\mathrm{MPa}$ ) and TBP plates (31.58 MPa).

For bone fragment displacement, similar bone fragment displacement results were observed in all groups. The maximum tibia fragment displacement was $0.16 \mathrm{~mm}$ in PPMT, $0.17 \mathrm{~mm}$ in PMT, and 0.12 $\mathrm{mm}$ in TBP. For the structural stiffness, a similar result was found for PPMT, PMT, and TBP. The stiffness was $1398.60 \mathrm{~N} / \mathrm{mm}$ in PPMT, $1459.80 \mathrm{~N} / \mathrm{mm}$ in PMT, and $1379.30 \mathrm{~N} / \mathrm{mm}$ in TBP.

\section{Discussion}

Posteromedial tibial plateau split fractures are common tibial plateau fractures. Approximately one third of bicondylar tibial plateau fractures have a posteromedial fragment in the coronal plane ${ }^{4}$. This is often caused by a high-energy injury mechanism. The mechanism involved in this fracture pattern may be one of knee flexion, knee varus, and internal medial femoral condyle rotation ${ }^{5}$. This type of fracture pattern is worth noting more than others affecting the tibial plateau because this fracture pattern easily causes instability within the knee joint. Previous studies presented that malalignment related to inadequate fixation and the associated soft tissue injuries were the two most important reasons for a poor prognosis $^{5-7}$.

The operative treatment goal for tibial plateau split fractures are anatomical reduction, especially in articular congruity restoration, stable fixation for early rehabilitation, and avoidance of complications, particularly infection and non-union. The tibial bone plate fixation is a major approach used to fix the fragment. Non-displaced posterior fracture fragments can usually be stabilized through the standard anterolateral approach. ${ }^{8,9}$ However, in the anteromedial approach ${ }^{10}$, the fracture site is shown from the lateral side. However, the medial collateral ligament (MCL) is easily injured during dissection. Therefore, a posteromedial approach is widely applied in the treatment of posterior medial condylar fractures. ${ }^{11,12}$ Satisfactory results have been achieved using this incision to expose the posterior medial condylar tibial plateau fracture. The posteromedial key fragment may displace distally and medially, especially when the knee is flexed. Several reports have illustrated the importance of coronal plane proximal tibial fractures, which are only visible on lateral radiographs or computed tomography scans. If displaced fractures in the 
coronal plane are not addressed, they may lead to the use of inappropriate fixation techniques. We have papers that proposed using a reverse L-shape incision that allows more space for reduction and easier implant placement. The T-shaped Buttress plate is a conventional implant for posteromedial tibial fracture fixation. Due to insufficient implant stability, nonunion would occur even using our direct approach and fixation. Therefore, a new implant design to provide enough stability that initiates bone union should be a better solution.

In this present study, three different proximate tibial bone plate designs were compared for stability after implantation. In the three plate fixations, an obvious stress concentration surrounding the screw holes was demonstrated. It is known that a smooth round hole in a plate causes a stress concentration. ${ }^{13}$ The highest peak von Mises stress occurred in the new designed plate. The possible reason might be the ovalshaped screw hole in this implant while the other two designs have a rounded screw hole. The peak von Mises stress among three plate fixations ranged from about 90 to about $110 \mathrm{MPa}$. There is a big gap between these values and the Titanium alloy fatigue strength (600 Mpa).${ }^{14}$ However, the commercialized T-Buttress plate is made of pure titanium. The fatigue strength of pure titanium is $230-280 \mathrm{MPa} .{ }^{15} \mathrm{We}$ can expect the T-Buttress plate would be at high risk of breakage. As for the screw stress distribution, the peak von Mises stress of the screw in all plate designs was almost twice that of the plate. However, the peak screw stress is much lower than the fatigue strength $(600 \mathrm{Mpa})^{14}$ and the yield strength of Titanium alloy (approximately $800 \mathrm{Mpa}$ ). ${ }^{16}$ These outcomes are in agreement with the in vitro experimental test ${ }^{17}$ that demonstrated no screw bending in a tibial plateau split fracture, even with loads as high as $900 \mathrm{~N}$. This study suggests no mechanical damage would be expected for the new design and the medial proximal tibial plate because of the simulated physiological load.

The axial construct stiffness from high to low is in the order of medial proximal tibial plate (1459.8 $\mathrm{N} / \mathrm{mm})$, the proximal posterior medial plate $(1398.6 \mathrm{~N} / \mathrm{mm})$ and the T-Buttress plate $(1379.3 \mathrm{~N} / \mathrm{mm})$ with the corresponding maximum axial displacement of $1.37,1.43,1.45 \mathrm{~mm}$, respectively. Direct comparisons of the calculated fragment movement with the experimental data reported in the literature are not appropriate. On one hand, there are limited experimental studies that focused on such comparisons. The found values in this study were similar with those reported by Zeng et al., ${ }^{18}$ who used synthetic bone femoral condyles to load tibia specimens with posteromedial tibial plateau split fracture. The measured fragment subsidence ranged from $0.832 \mathrm{~mm}$ for the T-shaped buttress plate to $1.559 \mathrm{~mm}$ for the lagscrews under $1500 \mathrm{~N}$ load. Nevertheless, they indicated that a posterior T-shaped buttress plate produced greater stability in controlling the posteromedial fragment movement than the medial dynamic compression plate and the lateral locking plate. We thought that the medial dynamic compression plate does not have fixed-angle stability while the medial plate and the proximal posterior medial plate involved in the current study both have a locking mechanism to improve the angular and axial stability for fracture fixation. Overall, the maximum fragment movement achieved in the three plate fixations were far below the fragment movement threshold usually considered clinically $(3 \mathrm{~mm})$ to evaluate the split tibial plateau fracture reduction success. ${ }^{18}$ 
In this study, the PPMT bone plate locking designs have batter stability than the conventional TBP plate. The features of this innovated PPMT bone plate include adequate stability for posteromedial fracture fixation, anatomical design that does not require plate bending for fitting. A conventional hole is located on the posteromedial fracture spike to allow buttressing. Locking designs for angular stability can be placed juxta-articular for subchondral fixation. Therefore, this innovative PPMT bone plate offers an alternative option for surgeons to treat posteromedial tibial plateau fractures.

Some limitations were inevitable. The fibula bone was not included. Only one type of posteromedial fracture was evaluated. Further studies should evaluate additional fracture types. The model used sustained only a static load. Nevertheless, the cyclic load usually occurs during daily activities. This study made use of known parameters and deduced information based on previous literature. Further studies using biomechanical testing models will be needed to establish this information to provide better accuracy.

\section{Conclusion}

This study investigated the structural stability of three different proximate tibial bone plate fixation designs for posteromedial tibial plateau split fractures. Previously, the traditional T-shaped buttress plate was considered using a commonly used implant that has superior structural stability to the traditional medial or lateral compression plate. The most important finding in this study is that the innovated proximal posterior medial tibia (PPMT) Locking Plate and proximal medial tibia (PMT) Locking Plate have comparable structural stability with the traditional TBP plate. The PPMT plate has multiple screw hole designs to offer more surgical implantation flexibility for surgeons. The plate placements for the PPMT and PMT are different. The surgeon could select the more appropriate bone plate to fixation according to different fracture modes.

\section{References}

1. Higgins, T. F., Kemper, D., \& Klatt, J. (2009). Incidence and morphology of the posteromedial fragment in bicondylar tibial plateau fractures. J Orthop Trauma, 23, 45-51

2. Ackerman, M. J., Spitzer, V. M., Scherzinger, A. L., \& Whitlock, D. G. (1995). The Visible Human data set: an image resource for anatomical visualization. Medinfo, 8(Pt 2), 1195-1198

3. Hsu, J. T., Chang, C. H., Huang, H. L., Zobitz, M. E., Chen, W. P., Lai, K. A., \& An, K. N. (2007). The number of screws, bone quality, and friction coefficient affect acetabular cup stability. Med Eng Phys, 29, 1089-1095

4. Barei, D. P., O'Mara, T. J., Taitsman, L. A., Dunbar, R. P., \& Nork, S. E. (2008). Frequency and fracture morphology of the posteromedial fragment in bicondylar tibial plateau fracture patterns. $J$ Orthop Trauma, 22, 176-182

5. Bhattacharyya, T., McCarty, L. P. 3rd, Harris, M. B., Morrison, S. M., Wixted, J. J., Vrahas, M. S., \& Smith, R. M. (2005). The posterior shearing tibial plateau fracture: treatment and results via a 
posterior approach. J Orthop Trauma, 19, 305-310

6. Luo, C. F., Jiang, R., Hu, C. F., \& Zeng, B. F. (2006). Medial double-plating for fracture dislocations involving the proximal tibia.. Knee, 13, 389-394

7. Boeck, H. D., \& Opdecam, P. (1995). Posteromedial tibial plateau fractures. Operative treatment by posterior approach. Clin Orthop Relat Res, 320, 125-128

8. Gösling, T., Schandelmaier, P., Marti, A., Hufner, T., Partenheimer, A., \& Krettek, C. (2004). Less invasive stabilization of complex tibial plateau fractures: a biomechanical evaluation of a unilateral locked screw plate and double plating. J Orthop Trauma, 18, 546-551

9. Cho, J. W., Kim, J., Cho, W. T., Kim, J. K., Samal, P., Gujjar, P. H. ... Oh, J. K. (2017). Approaches and fixation of the posterolateral fracture fragment in tibial plateau fractures: a review with an emphasis on rim plating via modified anterolateral approach. Int Orthop, 41, 1887-1897

10. Chen, H. W., Chen, C. Q., \& Yi, X. H. (2015). Posterior tibial plateau fracture: a new treatment-oriented classification and surgical management. Int J Clin Exp Med, 8, 472-479

11. Carlson, D. A. (2005). Posterior bicondylar tibial plateau fractures. J Orthop Trauma, 19, 73-78

12. Brunner, A., Honigmann, P., Horisberger, M., \& Babst, R. (2009). Open reduction and fixation of medial Moore type II fractures of the tibial plateau by a direct dorsal approach. Arch Orthop Trauma Surg, $129,1233-1238$

13. Pilkey, W. D. (1997). Peterson's stress concentration factors (2nd ed.). New York: Wiley

14. Toeh, S. H. (2000). Fatigue of biomaterials: a review. Int J Fatigue, 22, 825-837

15. Black, J. (Ed.). (1998). Handbook of biomaterials properties. part II. New York: Chapman and Hall

16. ASTM F136 Standard specification for wrought titanium-6aluminum-4vanadium ELI (extra low interstitial) alloy for surgical implant applications (UNS R56401).

17. Parker, P. J., Tepper, K. B., Brumback, R. J., Novak, V. P., \& Belkoff, S. M. (1999). Biomechanical comparison of fixation of type-I fractures of the lateral tibial plateau. Is the antiglide screw effective? $J$ Bone Joint Surg Br, 81, 478-480

18. Zeng, Z. M., Luo, C. F., Putnis, S., \& Zeng, B. F. (2011). Biomechanical analysis of posteromedial tibial plateau split fracture fixation. Knee, $18,51-54$

\section{Table}

Table 1

Maximum equivalent von mises stress (MPa) on PPMT, TBP, PMT on each screw. 


\begin{tabular}{|llllllll|}
\hline & Screw 1 & Screw 2 & Screw 3 & Screw 4 & Screw 5 & Screw 6 & Screw 7 \\
\hline PPMT & 297.22 & 69.92 & 167.79 & 129.96 & 121.25 & 93.97 & - \\
\hline TBP & 193.08 & 49.36 & 189.84 & 68.15 & 79.51 & 45.82 & - \\
\hline PMT & 205.53 & 85.79 & 119.09 & 85.16 & 96.82 & 98.95 & 110.39 \\
\hline
\end{tabular}

\section{Figures}

(a)

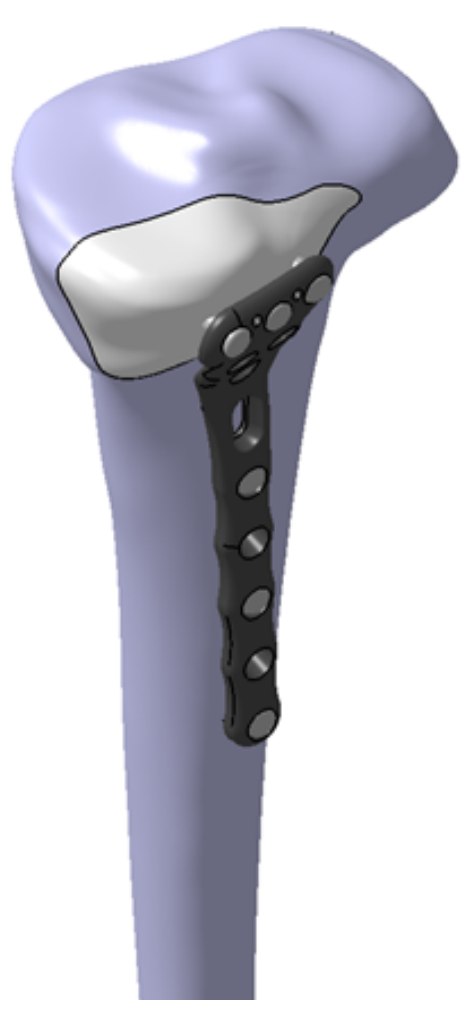

(b)

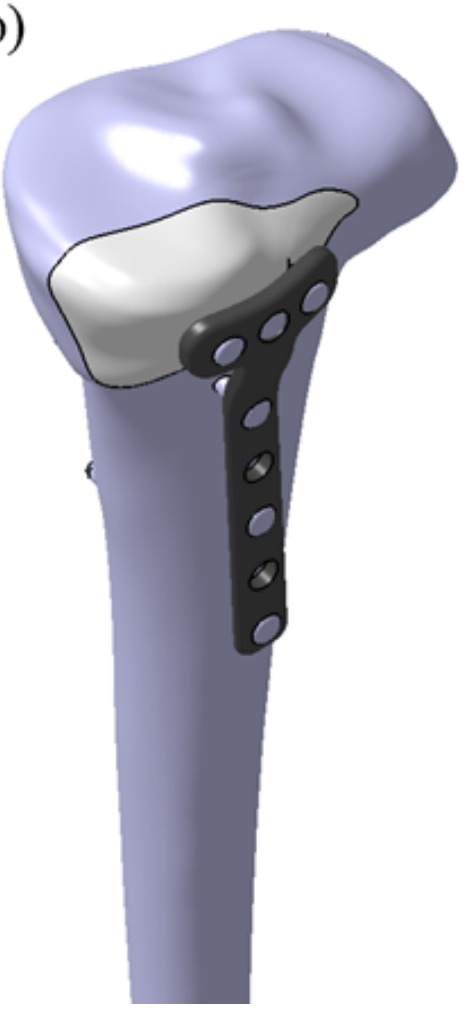

(c)

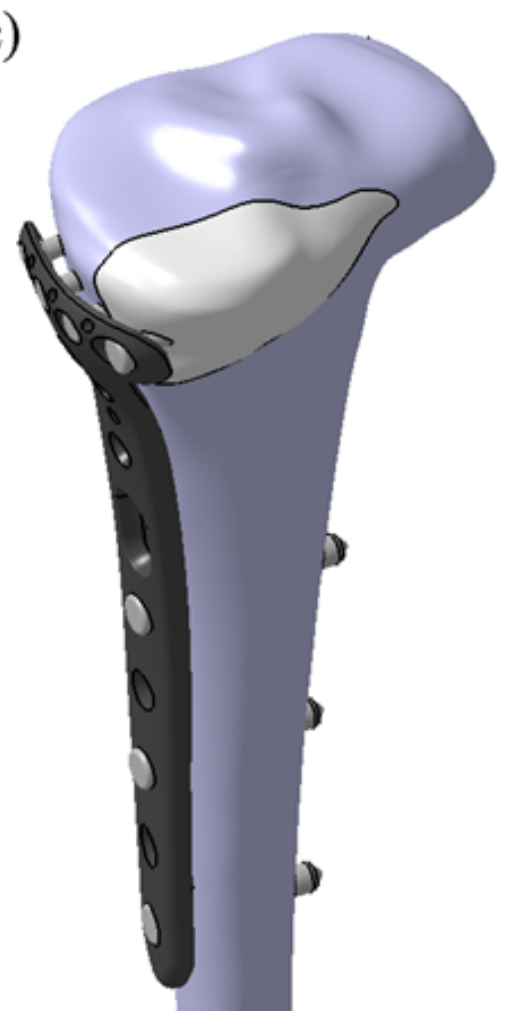

Figure 1

Three different type proximal tibia plates were implanted onto a proximal tibia (a) Proximal Posterior Medial Tibia Locking Plate System (PPMT), (b) T-shaped Buttress Plate (TBP), and (c) Proximal Medial Tibia Locking Plate System (PMT). 


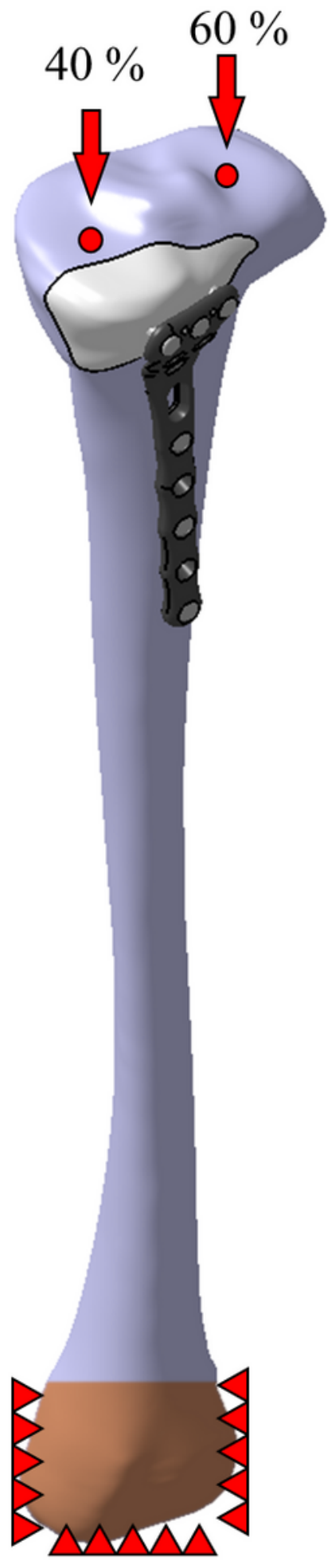

\section{Figure 2}

Load and boundary conditions in each group, the distal end of the tibia was fully fixed in all degrees of freedom; the load was applied to the proximal tibial plateau to simulate the single leg stand. 

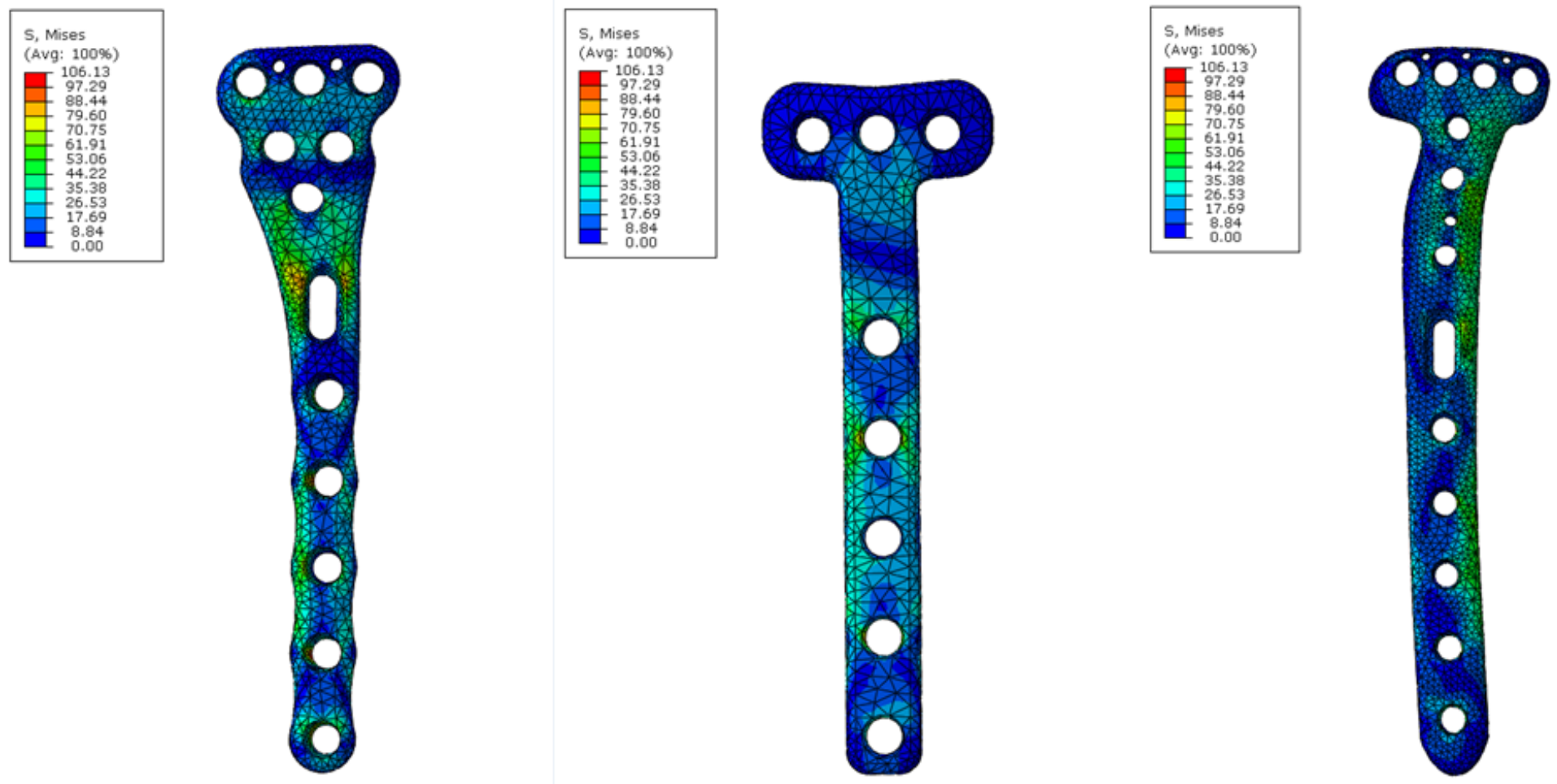

Figure 3

Equivalent Von Mises Stress (EVMS) distribution on three different type of proximal tibia plates; (a) Proximal Posterior Medial Tibia Locking Plate System (PPMT), (b) T-shaped Buttress Plate (TBP), and (c) Proximal Medial Tibia Locking Plate System (PMT).
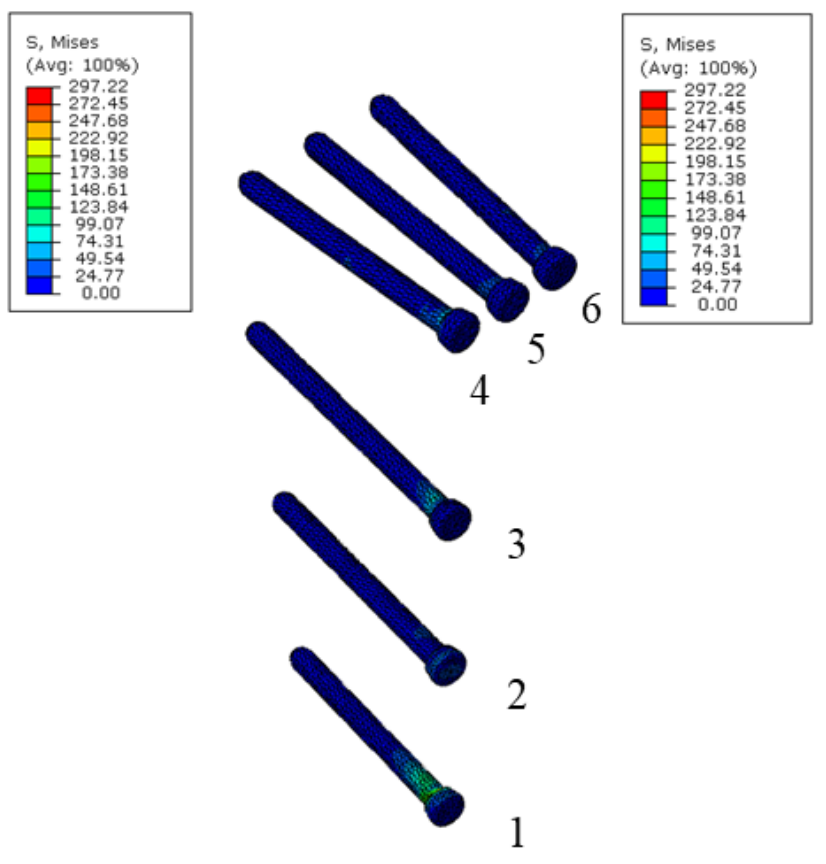

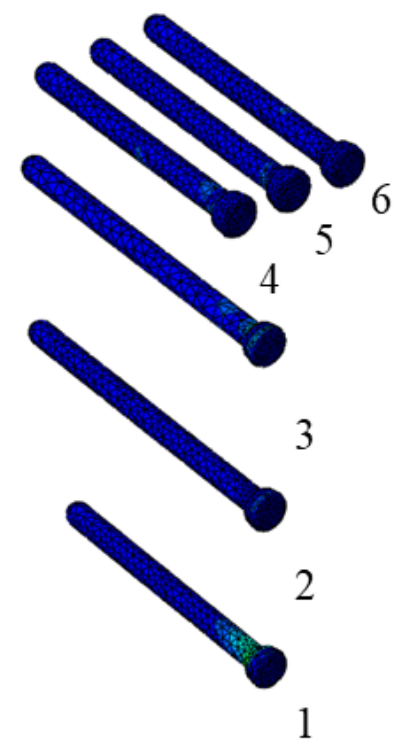

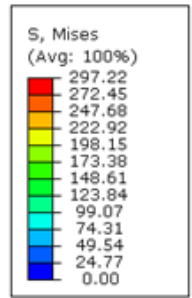
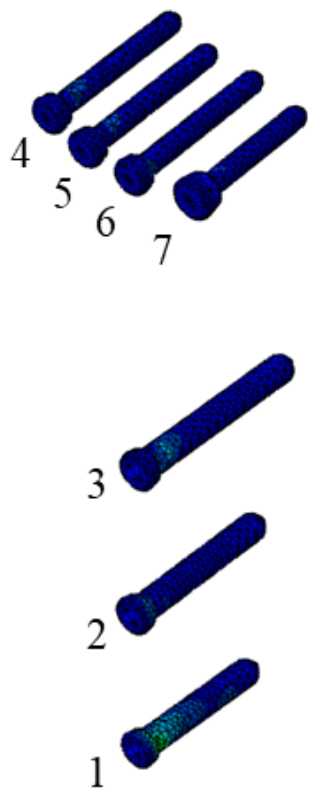

Figure 4 
Equivalent Von Mises Stress (EVMS) distribution on bone screws; (a) Proximal Posterior Medial Tibia Locking Plate System (PPMT), (b) T-shaped Buttress Plate (TBP), and (c) Proximal Medial Tibia Locking Plate System (PMT).
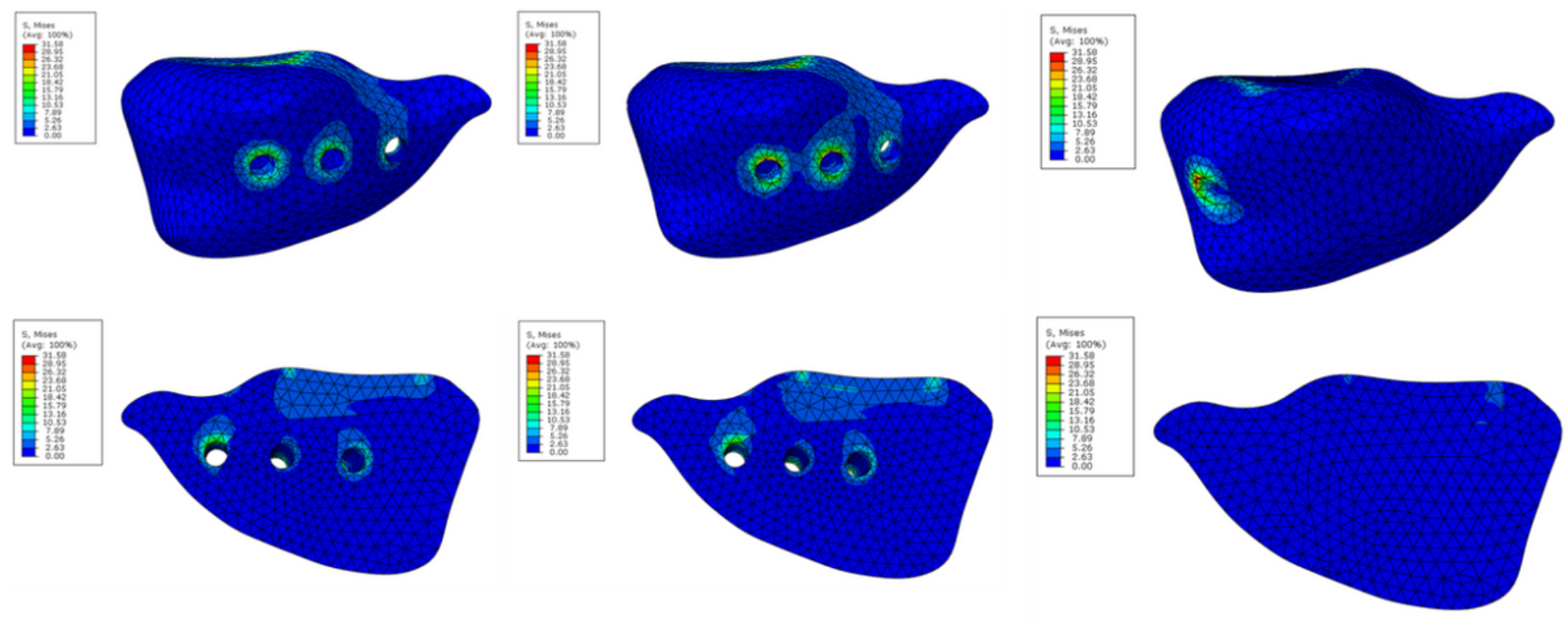

Figure 5

Equivalent Von Mises Stress (EVMS) distribution on posteromedial bone fragment; (a) Proximal Posterior Medial Tibia Locking Plate System (PPMT), (b) T-shaped Buttress Plate (TBP), and (c) Proximal Medial Tibia Locking Plate System (PMT). 